\title{
Diagnosis of Testicular Cancer via Point-of-Care Ultrasound
}

\author{
Roberto A. Velazquez-Amador, $\mathrm{MD}^{1}$; Verna Marquez, MD ${ }^{2}$; Rohit Kanda, MS-IV \\ ${ }^{1}$ Rio Bravo Family Medicine Residency Program, Bakersfield, CA \\ ${ }^{2}$ Rio Bravo Family Medicine Program, Bakersfield, CA \\ ${ }^{3}$ Ross University School of Medicine at Kern Medical Center, Bakersfield, CA
}

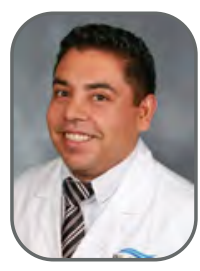

Roberto A. Velazquez-Amador, MD

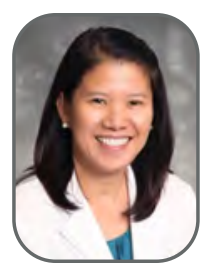

Verna Marquez, MD

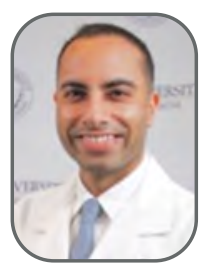

Rohit Kanda, MS-IV

\section{CORRESPONDENCE:}

Roberto A. Velazquez-Amador, MD mdrava@hotmail.com

DOI: $10.38206 / 140111$

\section{KEYWORDS:}

Embryonal Carcinoma, POC Ultrasonography, POCUS, Point-of-Care Ultrasound, Testicular Cancer

\section{ABSTRACT}

Point-of-care ultrasound is an emerging diagnostic tool in Family Medicine practice. The case presented in this article exemplifies how point-of-care ultrasound can facilitate diagnosis onsite, expedite care, and possibly improve prognosis.

\section{INTRODUCTION}

In this case report, a 23-year-old Hispanic male came to the office complaining of a lump on his right testicle. Using point-ofcare ultrasound (POCUS), an assessment was used to confirm the mass and expedite diagnoses and treatment.

\section{CASE PRESENTATION}

This is a 23-year-old Hispanic male patient resident of Bakersfield who was consulted via telemedicine. He complained about a right testicle lump that he had noticed for one month on selfexamination. He described it as solid and attached to the testicle with significant growth during this period to the size of a dime. The patient was immediately asked to come to the clinic to perform a point-of-care ultrasound. The POCUS showed a solid, non-vascular isoechoic to hypoechoic lobulated mass lesion measuring about $2.02 \times 1.5 \mathrm{~cm}$ in the right testis with internal non-shadowing hyperechoic areas (Figure 1), which was concerning for cancer.

The formal US showed a well-defined hypoechoic nodule in the right testicle measuring $2 \times 1.6 \mathrm{~cm}$, consistent with testicular carcinoma. A referral to Urology was placed. CT of the abdomen did not show any evidence of metastatic disease in the abdomen or pelvis. No retroperitoneal or pelvic lymphadenopathy. AFP was $5.1(<6.1)$, HCG 6 (5), and LDH was not performed. The patient underwent right radical inguinal orchiectomy, and pathology reported: Embryonal carcinoma, $2 \mathrm{~cm}$, pT1a, no LVI, margins were clear. The patient recovered well from surgery. He was referred for adjuvant chemotherapy and one BEP cycle, given that this is a pure embryonal carcinoma. Again, the patient recovered, and surveillance with tumor markers continued per protocol. 


\section{CONCLUSION}

POCUS is a time-efficient tool that allows medical practices to diagnose pathologies that otherwise would have to be found on formal imaging. This allows the provider to make an immediate decision and expedite care. Also, POCUS allows the medical practitioner to identify in real-time the suspected diagnosis.

We emphasize that a cursory point-of-care ultrasound is really an extension of the physical examination (like a stethoscope or sphygmomanometer) and does not obviate the necessity of a complete comprehensive ultrasonogram performed by an ultrasonographer and interpreted by a radiologist or ultrasonologist in a radiology department dedicated to medical imaging. These ultrasonograms are much more complete, supervised by a radiologist, and image elements that a family medicine physician may not know about, observe, not report.

\section{FIGURE 1.}

Results of the POCUS

Right testicle mass measuring $2.02 \times 1.5 \mathrm{~cm}$ (LxH). Right testicle (on right) measures $4.2 \times 1.84 \mathrm{~cm}(\mathrm{LxH})$

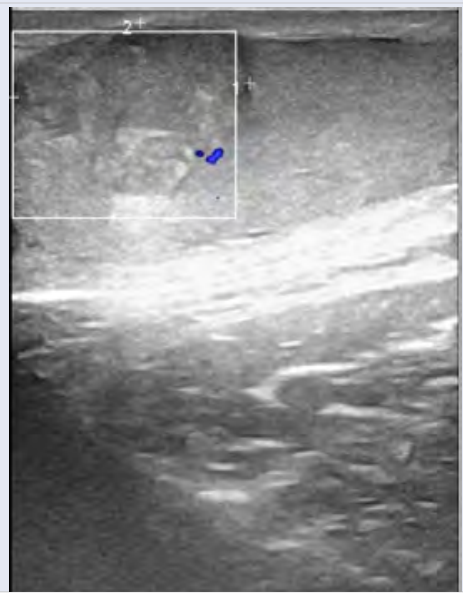

AUTHOR DISCLOSURES

No relevant financial affiliations or conflicts of interest.

\section{CONGRATULATIONS TO SOUTHERN CALIFORNIA CLINICIANS}

$A B C$ Pharmacy

\section{California Locations}

\section{LAGUNA HILLS \\ 23141 Moulton Pkwy. \\ 949-916-9990}

MONTCLAIR

5050 San Bernardino St., \#110

909-264-1331

ONTARIO

2920 Inland Empire \#112

909-291-3800
RANCHO CUCAMONGA

7974 Haven Ave., \#120

909-285-1118

RIALTO

826 East Foothill Blvd.

909-546-1000

REDLANDS

1600 E Citrus Ave.

909-726-3080
RIVERSIDE

777 W Blaine St.

951-299-9922

UPLAND

954 W Foothill Blvd., \#D

909-946-5512 